\title{
ONE GENERALIZED INEQUALITY FOR CONVEX FUNCTIONS ON THE TRIANGLE
}

\section{ZLATKO PAVIĆ}

Mechanical Engineering Faculty in Slavonski Brod

University of Osijek

Trg Ivane Brlić Mažuranić 2

35000 Slavonski Brod

Croatia

e-mail: zlatko.pavic@sfsb.hr

\begin{abstract}
The aim of the paper is to obtain a very general inequality for convex functions on the triangle. To construct such inequality, we include positive linear functionals on the space of real functions. That inequality can be applied to various forms of mathematical means, and its integral form contains the Jensen, Fejér and Hermite-Hadamard inequality.
\end{abstract}

\section{Introduction}

\subsection{Convex functions on the triangle}

The triangle with vertices $a, b, c \in \mathbb{R}^{2}$ that do not belong to the same line is used permanently throughout the article. It will be marked by $\Delta$ or $a b c$, and its interior will be marked by $\Delta^{\circ}$. Each point $x \in \Delta$ can be represented by the unique trinomial convex combination

2010 Mathematics Subject Classification: 26D15, 26B25, 46N10.

Keywords and phrases: convex function, triangle, space of real functions, positive linear functional.

Received August 23, 2015

(c) 2015 Scientific Advances Publishers 


$$
x=\alpha a+\beta b+\gamma c
$$

The coefficients can be expressed by the ratio of areas

$$
\alpha=\frac{\operatorname{ar}(x b c)}{\operatorname{ar}(a b c)}, \quad \beta=\frac{\operatorname{ar}(x a c)}{\operatorname{ar}(a b c)}, \quad \gamma=\frac{\operatorname{ar}(x a b)}{\operatorname{ar}(a b c)},
$$

and thus by the ratio of determinants

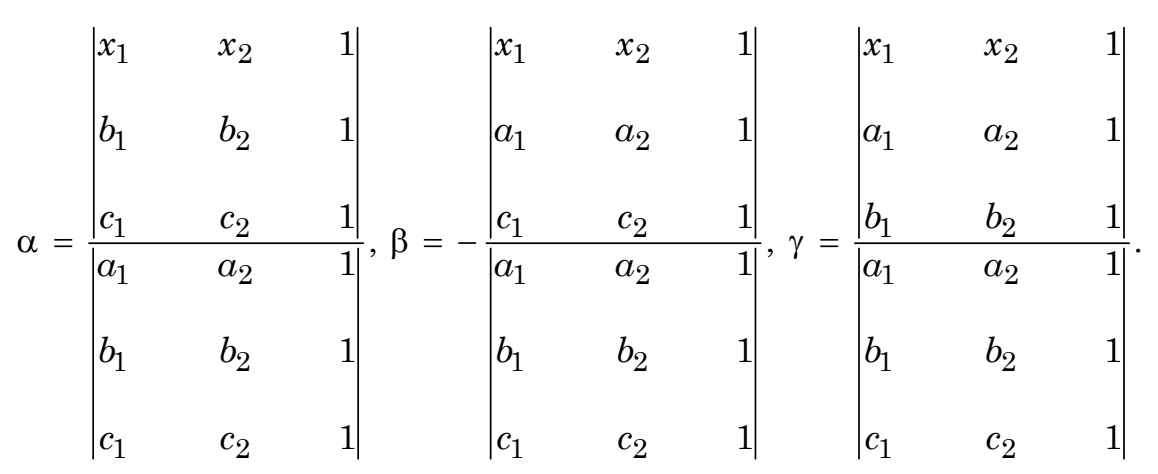

Let $f: \Delta \rightarrow \mathbb{R}$ be a convex function. The secant plane of $f$ passes through the respective graph points of $a, b$, and $c$, and its equation is

$$
f_{a b c}^{\mathrm{sec}}(x)=\frac{\operatorname{ar}(x b c)}{\operatorname{ar}(a b c)} f(a)+\frac{\operatorname{ar}(x a c)}{\operatorname{ar}(a b c)} f(b)+\frac{\operatorname{ar}(x a b)}{\operatorname{ar}(a b c)} f(c) .
$$

Let $d \in \Delta^{\circ}$ be a point of the triangle interior. The support planes of $f$ at $d$ pass through the respective graph point of $d$, and their equations depend on the pairs of slope coefficients $\kappa_{1} \in\left[f_{1}^{\prime}(d-), f_{1}^{\prime}(d+)\right]$ and $\kappa_{2} \in\left[f_{2}^{\prime}(d-), f_{2}^{\prime}(d+)\right]$. For the specified pair of coefficients $\kappa_{1}$ and $\kappa_{2}$, the corresponding equation is

$$
f_{d}^{\sup }\left(x_{1}, x_{2}\right)=\kappa_{1}\left(x_{1}-d_{1}\right)+\kappa_{2}\left(x_{2}-d_{2}\right)+f\left(d_{1}, d_{2}\right) .
$$

The support-secant plane inequality

$$
f_{d}^{\sup }(x) \leq f(x) \leq f_{a b c}^{\mathrm{sec}}(x)
$$

holds for every $x \in \Delta$. The secant and support plane of a convex surface are presented geometrically in Figure 1. 


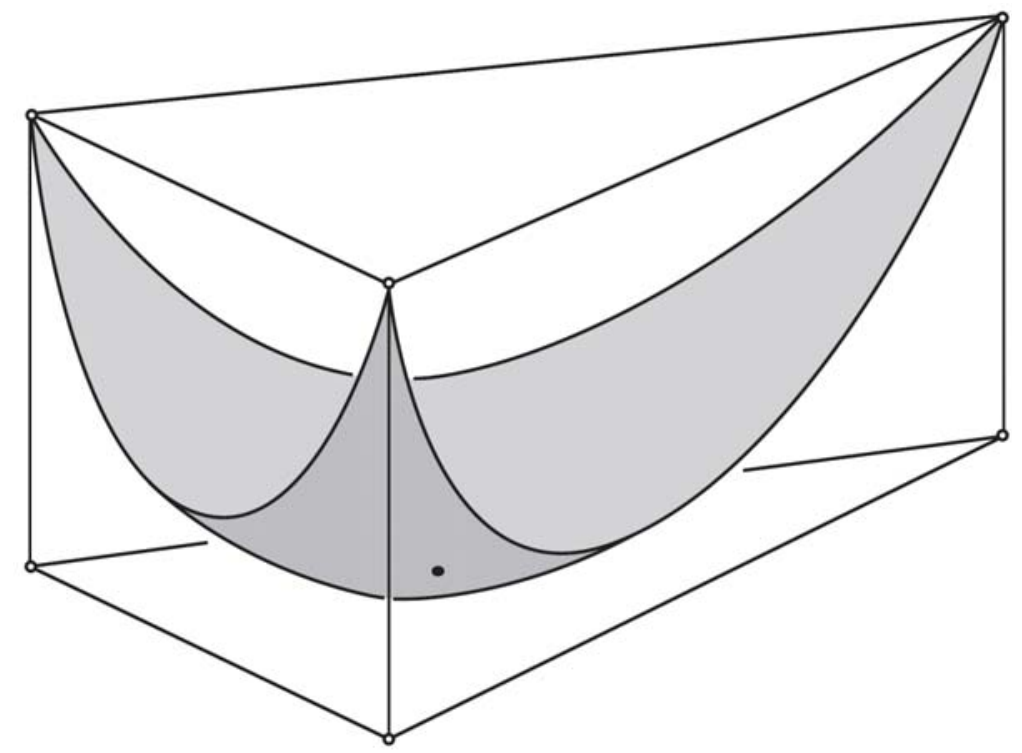

Figure 1. Secant and support plane of a convex function.

\subsection{Positive linear functionals on the space of real functions}

Let $X$ be a nonempty set, and let $\mathbb{F}=\mathbb{F}(X)$ be a subspace of the linear space of all real functions on the domain $X$. We assume that the space $\mathbb{F}$ contains the unit function $u$ defined by $u(x)=1$ for every $x \in X$. Then, the space $\mathbb{F}$ contains every real constant $\kappa$ within the meaning of $\kappa=\kappa u$, and $\mathbb{F}$ contains every composite function $f\left(g_{1}, g_{2}\right)$ of an affine function $f: \mathbb{R}^{2} \rightarrow \mathbb{R}$ and a pair of functions $g_{1}, g_{2} \in \mathbb{F}$. Specifically, using the equation $f\left(x_{1}, x_{2}\right)=\kappa_{1} x_{1}+\kappa_{2} x_{2}+\kappa_{3}$, we have the composition

$$
f\left(g_{1}, g_{2}\right)=\kappa_{1} g_{1}+\kappa_{2} g_{2}+\kappa_{3} u
$$

which belongs to the space $\mathbb{F}$.

Let $\mathbb{L}=\mathbb{L}(\mathbb{F}(X))$ be the space of all linear functionals on the space $\mathbb{F}(X)$. A functional $L \in \mathbb{L}$ is said to be positive (nonnegative) if the inequality $L(g) \geq 0$ holds for every nonnegative function $g \in \mathbb{F}$. If 
$g_{1}, g_{2} \in \mathbb{F}$ are functions such that $g_{1}(x) \leq g_{2}(x)$ for $x \in X$, then a positive functional $L$ satisfies the inequality

$$
L\left(g_{1}\right) \leq L\left(g_{2}\right) .
$$

A functional $L \in \mathbb{L}$ is said to be unital (normalized) if $L(u)=1$. Such functional has the property $L(\kappa u)=\kappa$ for every real constant $\kappa$.

\section{Main Results}

The first lemma provides a basic inclusion relating to the image of a pair of functions $g_{1}, g_{2} \in \mathbb{F}$. The proof of lemma includes a convex analytics through the application of convex combinations.

Lemma 2.1. Let $g_{1}, g_{2} \in \mathbb{F}$ be functions such that $\left(g_{1}(x), g_{2}(x)\right) \in \Delta$ for $x \in X$.

Then a positive unital functional $L \in \mathbb{L}$ satisfies the inclusion

$$
\left(L\left(g_{1}\right), L\left(g_{2}\right)\right) \in \Delta .
$$

Proof. Taking $x$ in $X$, we have the point $\left(g_{1}(x), g_{2}(x)\right)$ in $\Delta$, and its unique convex combination

$$
\left(g_{1}(x), g_{2}(x)\right)=\alpha(x)\left(a_{1}, a_{2}\right)+\beta(x)\left(b_{1}, b_{2}\right)+\gamma(x)\left(c_{1}, c_{2}\right) .
$$

Using equations in (3), we can determine functions $\alpha(x), \beta(x)$, and $\gamma(x)$ showing that they belong to $\mathbb{F}$. For example, $\alpha(x)=\alpha_{1} g_{1}(x)+\alpha_{2} g_{2}(x)+\alpha_{3}$. Since the functional $L$ is positive, the numbers $L(\alpha), L(\beta)$, and $L(\gamma)$ are nonnegative, and since $\alpha(x)+\beta(x)+\gamma(x)=u(x)$, it follows that $L(\alpha)+L(\beta)+L(\gamma)=1$.

Acting with the functional $L$ to each of the coordinates of equation in (10), we obtain the convex combination

$$
\left(L\left(g_{1}\right), L\left(g_{2}\right)\right)=L(\alpha)\left(a_{1}, a_{2}\right)+L(\beta)\left(b_{1}, b_{2}\right)+L(\gamma)\left(c_{1}, c_{2}\right),
$$

ensuring that the point $\left(L\left(g_{1}\right), L\left(g_{2}\right)\right)$ belongs to the triangle $\Delta$. 
Using hyperplanes, McShane proved that the inclusion in (9) generally applies to closed convex sets in $\mathbb{R}^{2}$. That part of his work (see [7]) he called the geometric formulation of Jensen's inequality.

The second lemma provides a basic equality relating to an affine function.

Lemma 2.2. Let $g_{1}, g_{2} \in \mathbb{F}$ be functions and $L \in \mathbb{L}$ be a unital functional.

Then an affine function $f: \mathbb{R}^{2} \rightarrow \mathbb{R}$ satisfies the equality

$$
f\left(L\left(g_{1}\right), L\left(g_{2}\right)\right)=L\left(f\left(g_{1}, g_{2}\right)\right) .
$$

Proof. Using the affine equation $f\left(x_{1}, x_{2}\right)=\kappa_{1} x_{1}+\kappa_{2} x_{2}+\kappa_{3}$, and applying the unital property of $L$, we obtain

$$
\begin{aligned}
f\left(L\left(g_{1}\right), L\left(g_{2}\right)\right) & =\kappa_{1} L\left(g_{1}\right)+\kappa_{2} L\left(g_{2}\right)+\kappa_{3} \\
& =L\left(\kappa_{1} g_{1}+\kappa_{2} g_{2}+\kappa_{3} u\right) \\
& =L\left(f\left(g_{1}, g_{2}\right)\right)
\end{aligned}
$$

proving the equality in formula (12).

Now we can include a continuous convex function, and get the main result.

Theorem 2.3. Let $g_{1}, g_{2} \in \mathbb{F}$ be functions such that $\left(g_{1}(x), g_{2}(x)\right) \in \Delta$ for $x \in X$. Let $L \in \mathbb{L}$ be a positive unital functional.

Then a continuous convex function $f: \Delta \rightarrow \mathbb{R}$ satisfies the double inequality

$$
f\left(L\left(g_{1}\right), L\left(g_{2}\right)\right) \leq L\left(f\left(g_{1}, g_{2}\right)\right) \leq f_{\Delta}^{\mathrm{sec}}\left(L\left(g_{1}\right), L\left(g_{2}\right)\right),
$$

if provided that the composite function $f\left(g_{1}, g_{2}\right)$ is in $\mathbb{F}$.

Proof. The point $l=\left(L\left(g_{1}\right), L\left(g_{2}\right)\right)$ belongs to the triangle $\Delta$ by Lemma 2.1. We sketch the proof in three steps depending on the position of $l$. 
If $l \in \Delta^{\circ}$, we take any support plane of $f$ at $l$. Acting with the positive functional $L$ to the following subinequality of the support-secant inequality in formula (6):

$$
f_{l}^{\text {sup }}\left(g_{1}(x), g_{2}(x)\right) \leq f\left(g_{1}(x), g_{2}(x)\right) \leq f_{\Delta}^{\text {sec }}\left(g_{1}(x), g_{2}(x)\right)
$$

we obtain

$$
L\left(f_{l}^{\mathrm{sup}}\left(g_{1}, g_{2}\right)\right) \leq L\left(f\left(g_{1}, g_{2}\right)\right) \leq L\left(f_{\Delta}^{\mathrm{sec}}\left(g_{1}, g_{2}\right)\right)
$$

Applying Lemma 2.2 to affine functions $f_{l}^{\text {sup }}$ and $f_{\Delta}^{\text {sec }}$, and putting $l$ instead of $\left(L\left(g_{1}\right), L\left(g_{2}\right)\right)$, the above inequality takes the form

$$
f_{l}^{\sup }\left(L\left(g_{1}\right), L\left(g_{2}\right)\right) \leq L\left(f\left(g_{1}, g_{2}\right)\right) \leq f_{\Delta}^{\mathrm{sec}}\left(L\left(g_{1}\right), L\left(g_{2}\right)\right),
$$

where the first term

$$
f_{l}^{\sup }\left(L\left(g_{1}\right), L\left(g_{2}\right)\right)=f\left(L\left(g_{1}\right), L\left(g_{2}\right)\right)
$$

If $l$ belongs to the relative interior of some side, we apply the previous procedure to the corresponding convex curve by using any support line relating to $l$, and the secant line relating to endpoints of that side.

If $l \in\{a, b, c\}$, we rely on the continuity of $f$ using a support plane at a point of the interior $\Delta^{\circ}$ that is close enough to $l$.

Formula (14) can be expressed in the form that includes the convex combination of the triangle vertices $a, b$, and $c$. The respective form of Theorem 2.3 is as follows:

Corollary 2.4. Let $g_{1}, g_{2} \in \mathbb{F}$ be functions such that $\left(g_{1}(x), g_{2}(x)\right) \in \Delta$ for $x \in X$. Let $L \in \mathbb{L}$ be a positive unital functional, and let $\alpha a+\beta b+\gamma c$ be the convex combination that is equal to $\left(L\left(g_{1}\right), L\left(g_{2}\right)\right)$. 
Then a continuous convex function $f: \Delta \rightarrow \mathbb{R}$ satisfies the double inequality

$$
f(\alpha a+\beta b+\gamma c) \leq L\left(f\left(g_{1}, g_{2}\right)\right) \leq \alpha f(a)+\beta f(b)+\gamma f(c),
$$

if provided that the composite function $f\left(g_{1}, g_{2}\right)$ is in $\mathbb{F}$.

Proof. The corresponding terms of formulae (14) and (15) are the same. As regards the last terms, we have

$$
f_{\Delta}^{\mathrm{sec}}\left(L\left(g_{1}\right), L\left(g_{2}\right)\right)=\alpha f_{\Delta}^{\mathrm{sec}}(a)+\beta f_{\Delta}^{\mathrm{sec}}(b)+\gamma f_{\Delta}^{\mathrm{sec}}(c)=\alpha f(a)+\beta f(b)+\gamma f(c)
$$

because of the affinity of $f_{\Delta}^{\text {sec }}$, and its coincidence with $f$ at vertices.

As for the coefficients in formula (15), let $l=\left(L\left(g_{1}\right), L\left(g_{2}\right)\right)$, and let $\Delta_{1}=l b c, \Delta_{2}=l a c, \Delta_{3}=l a b$. Putting $x=l$ in formula (2), we get

$$
\alpha=\frac{\operatorname{ar}\left(\Delta_{1}\right)}{\operatorname{ar}(\Delta)}, \quad \beta=\frac{\operatorname{ar}\left(\Delta_{2}\right)}{\operatorname{ar}(\Delta)}, \quad \gamma=\frac{\operatorname{ar}\left(\Delta_{3}\right)}{\operatorname{ar}(\Delta)}
$$

Functional inequalities for functions that are not necessarily convex were considered in [5].

\section{Applications to Discrete and Integral Inequalities}

We utilize Theorem 2.3 to derive the generalizations of important inequalities for convex functions on the triangle.

The following is the discrete version of Theorem 2.3.

Corollary 3.1. Let $g_{1}, g_{2}: \Delta \rightarrow \mathbb{R}$ be functions such that $\left(g_{1}(x)\right.$, $\left.g_{2}(x)\right) \in \Delta$ for $x \in \Delta$, and let $h: \Delta \rightarrow \mathbb{R}$ be a positive function. Let $x_{1}, \ldots, x_{n} \in \Delta$ be points, let

$$
l=\left(\frac{\sum_{i=1}^{n} g_{1}\left(x_{i}\right) h\left(x_{i}\right)}{\sum_{i=1}^{n} h\left(x_{i}\right)}, \frac{\sum_{i=1}^{n} g_{2}\left(x_{i}\right) h\left(x_{i}\right)}{\sum_{i=1}^{n} h\left(x_{i}\right)}\right)
$$

and let $\Delta_{1}=l b c, \Delta_{2}=l a c, \Delta_{3}=l a b$. 
Then a continuous convex function $f: \Delta \rightarrow \mathbb{R}$ satisfies the double inequality

$$
\begin{gathered}
f\left(\frac{\sum_{i=1}^{n} g_{1}\left(x_{i}\right) h\left(x_{i}\right)}{\sum_{i=1}^{n} h\left(x_{i}\right)}, \frac{\sum_{i=1}^{n} g_{2}\left(x_{i}\right) h\left(x_{i}\right)}{\sum_{i=1}^{n} h\left(x_{i}\right)}\right) \leq \frac{\sum_{i=1}^{n} f\left(g_{1}\left(x_{i}\right), g_{2}\left(x_{i}\right)\right) h\left(x_{i}\right)}{\sum_{i=1}^{n} h\left(x_{i}\right)} \\
\leq \frac{\operatorname{ar}\left(\Delta_{1}\right)}{\operatorname{ar}(\Delta)} f(a)+\frac{\operatorname{ar}\left(\Delta_{2}\right)}{\operatorname{ar}(\Delta)} f(b)+\frac{\operatorname{ar}\left(\Delta_{3}\right)}{\operatorname{ar}(\Delta)} f(c)
\end{gathered}
$$

Proof. Let $\mathbb{F}$ be the space of all real functions on the domain $X=\Delta$. We take the summarizing linear functional defined by

$$
L(q)=L(q ; h)=\frac{\sum_{i=1}^{n} q_{i}\left(x_{i}\right) h_{i}\left(x_{i}\right)}{\sum_{i=1}^{n} h_{i}\left(x_{i}\right)}
$$

for every function $q \in \mathbb{F}$. The summarizing functional $L$ is positive and unital.

Applying $L$ to the pair of given functions $g_{1}$ and $g_{2}$, we get the point

$$
l=\left(L\left(g_{1}\right), L\left(g_{2}\right)\right)=\left(\frac{\sum_{i=1}^{n} g_{1}\left(x_{i}\right) h\left(x_{i}\right)}{\sum_{i=1}^{n} h\left(x_{i}\right)}, \frac{\sum_{i=1}^{n} g_{2}\left(x_{i}\right) h\left(x_{i}\right)}{\sum_{i=1}^{n} h\left(x_{i}\right)}\right),
$$

and applying $L$ to the composition $f\left(g_{1}, g_{2}\right)$, we get the sums ratio

$$
L\left(f\left(g_{1}, g_{2}\right)\right)=\frac{\sum_{i=1}^{n} f\left(g_{1}\left(x_{i}\right), g_{2}\left(x_{i}\right)\right) h\left(x_{i}\right)}{\sum_{i=1}^{n} h\left(x_{i}\right)}
$$

Using $x=\left(L\left(g_{1}\right), L\left(g_{2}\right)\right)$ in the equation of the secant plane in formula (4), we get

$$
f_{\Delta}^{\mathrm{sec}}\left(L\left(g_{1}\right), L\left(g_{2}\right)\right)=\frac{\operatorname{ar}\left(\Delta_{1}\right)}{\operatorname{ar}(\Delta)} f(a)+\frac{\operatorname{ar}\left(\Delta_{2}\right)}{\operatorname{ar}(\Delta)} f(b)+\frac{\operatorname{ar}\left(\Delta_{3}\right)}{\operatorname{ar}(\Delta)} f(c) .
$$


Embedding the right-hand sides of Equations (20), (21), and (22) into the inequality in formula (14), we obtain the discrete inequality in formula (18).

The next is the integral version of Theorem 2.3.

Corollary 3.2. Let $g_{1}, g_{2}: \Delta \rightarrow \mathbb{R}$ be integrable functions such that $\left(g_{1}(x), g_{2}(x)\right) \in \Delta$ for $x \in \Delta$, and let $h: \Delta \rightarrow \mathbb{R}$ be a positive integrable function. Let

$$
l=\left(\frac{\int_{\Delta} g_{1}(x) h(x) d x}{\int_{\Delta} h(x) d x}, \frac{\int_{\Delta} g_{2}(x) h(x) d x}{\int_{\Delta} h(x) d x}\right),
$$

and let $\Delta_{1}=l b c, \Delta_{2}=l a c, \Delta_{3}=l a b$.

Then a continuous convex function $f: \Delta \rightarrow \mathbb{R}$ satisfies the double inequality

$$
\begin{gathered}
f\left(\frac{\int_{\Delta} g_{1}(x) h(x) d x}{\int_{\Delta} h(x) d x}, \frac{\int_{\Delta} g_{2}(x) h(x) d x}{\int_{\Delta} h(x) d x}\right) \leq \frac{\int_{\Delta} f\left(g_{1}(x), g_{2}(x)\right) h(x) d x}{\int_{\Delta} h(x) d x} \\
\leq \frac{\operatorname{ar}\left(\Delta_{1}\right)}{\operatorname{ar}(\Delta)} f(a)+\frac{\operatorname{ar}\left(\Delta_{2}\right)}{\operatorname{ar}(\Delta)} f(b)+\frac{\operatorname{ar}\left(\Delta_{3}\right)}{\operatorname{ar}(\Delta)} f(c) .
\end{gathered}
$$

Proof. Let $\mathbb{F}$ be the space of all integrable functions over the domain $X=\Delta$. According to the Lebesgue theorem on the Riemann integral, the composition $f\left(g_{1}, g_{2}\right)$ is integrable over $\Delta$ because is bounded, and continuous almost everywhere in $\Delta$.

We take the integrating linear functional defined by

$$
L(q)=L(q ; h)=\frac{\int_{\Delta} q(x) h(x) d x}{\int_{\Delta} h(x) d x},
$$

for every function $q \in \mathbb{F}$, and further follow the proof of Corollary 3.1. 
The inequality in formula (24) contains the extended integral form of Jensen's inequality (see [4]), the Fejér inequality (see [1]), and consequently the Hermite-Hadamard inequality (see [3] and [2]). The inequality simplifications are as follows.

Using the unit function $h\left(x_{1}, x_{2}\right)=1$, in which case

$$
l=\left(\frac{\int_{\Delta} g_{1}(x) d x}{\operatorname{ar}(\Delta)}, \frac{\int_{\Delta} g_{2}(x) d x}{\operatorname{ar}(\Delta)}\right)
$$

we get the extended integral form of Jensen's inequality

$$
\begin{gathered}
f\left(\frac{\int_{\Delta} g_{1}(x) d x}{\operatorname{ar}(\Delta)}, \frac{\int_{\Delta} g_{2}(x) d x}{\operatorname{ar}(\Delta)}\right) \leq \frac{\int_{\Delta} f\left(g_{1}(x), g_{2}(x)\right) d x}{\operatorname{ar}(\Delta)} \\
\quad \leq \frac{\operatorname{ar}\left(\Delta_{1}\right)}{\operatorname{ar}(\Delta)} f(a)+\frac{\operatorname{ar}\left(\Delta_{2}\right)}{\operatorname{ar}(\Delta)} f(b)+\frac{\operatorname{ar}\left(\Delta_{3}\right)}{\operatorname{ar}(\Delta)} f(c) .
\end{gathered}
$$

Now we use the projections $g_{1}\left(x_{1}, x_{2}\right)=x_{1}$ and $g_{2}\left(x_{1}, x_{2}\right)=x_{2}$, and a positive function $h\left(x_{1}, x_{2}\right)$ whose barycenter $l$ falls into $(a+b+c) / 3$. Thus

$$
l=\left(\frac{\int_{\Delta} x_{1} h(x) d x}{\int_{\Delta} h(x) d x}, \frac{\int_{\Delta} x_{2} h(x) d x}{\int_{\Delta} h(x) d x}\right)=\frac{a+b+c}{3},
$$

indicating that $\alpha=\beta=\gamma=1 / 3$, and we have the Fejér inequality

$$
f\left(\frac{a+b+c}{3}\right) \leq \frac{\int_{\Delta} f(x) h(x) d x}{\int_{\Delta} h(x) d x} \leq \frac{f(a)+f(b)+f(c)}{3} .
$$


Putting the unit function $h\left(x_{1}, x_{2}\right)=1$ in Fejér's inequality in formula (29), we obtain the Hermite-Hadamard inequality

$$
f\left(\frac{a+b+c}{3}\right) \leq \frac{\int_{\Delta} f(x) d x}{\operatorname{ar}(\Delta)} \leq \frac{f(a)+f(b)+f(c)}{3} .
$$

Improvements of the Hermite-Hadamard inequality for convex functions on the bounded closed interval of real numbers were considered in [6].

\section{Acknowledgements}

The author would like to thank Velimir Pavić who has prepared Figure 1.

\section{References}

[1] L. Fejér, Über die Fourierreihen II, Math. Naturwiss. Anz. Ungar. Akad. Wiss. 24 (1906), 369-390.

[2] J. Hadamard, Étude sur les propriétés des fonctions entières et en particulier d'une fonction considerée par Riemann, J. Math. Pures Appl. 58 (1893), 171-215.

[3] Ch. Hermite, Sur deux limites d'une intégrale définie, Mathesis 3 (1883), 82.

[4] J. L. W. V. Jensen, Sur les fonctions convexes et les inégalités entre les valeurs moyennes, Acta Math. 30 (1906), 175-193.

[5] Z. Pavić, Functions like convex functions, J. Funct. Spaces 2015 (2015), Article ID 919470.

[6] Z. Pavić, Improvements of the Hermite-Hadamard inequality, J. Inequal. Appl. 2015 (2015), Article ID 222.

[7] E. J. McShane, Jensen's inequality, Bull. Amer. Math. Soc. 43 (1937), 521-527. 